\title{
Research on Passive Troposcatter Location System
}

\author{
Zan LIU, Xihong CHEN, Qiang LIU \\ Air and Missile Defence College, Air Force Engineering University, 710051, Xi'an, People's Republic of China \\ kgdliuzan@163.com, xhchen0315217@163.com,dreamlq@163.com
}

Submitted June 10, 2018 / Accepted January 7, 2019

\begin{abstract}
Electromagnetic (EM) wave of enemy radar propagated by troposcatter can be utilized for beyond lineof-sight (b-LoS) location. To provide theoretical basis for passive troposcatter location system, channel characteristics including propagation loss, refraction effect and multipath fading are analyzed. Ray tracing method improved by tropospheric model is employed to describe the refraction effect. Correlation coefficient on the basis of transfer function is deduced to evaluate multipath fading. According to troposcatter characteristics, maximum operating range is estimated, cooperative energy detection is introduced to cope with the received signal without priori knowledge. The principle and accuracy of location algorithm based on azimuth are researched. Spatial smoothing is introduced under the situation that uncorrelated and coherent signals coexist. Through analyzing consequences, future directions are suggested.
\end{abstract}

\section{Keywords}

Passive troposcatter location system, channel characteristics, signal detection, location algorithm, direction-of-arrival (DOA)

\section{Introduction}

Locating enemy radar plays an important role in the modern warfare. Satellite system can realize beyond lineof-sight (b-LoS) location [1]. However, satellite link has security problems and can be interrupted by hostile jamming. Major radars employ the electromagnetic (EM) wave ranging in microwave band to detect and track their targets. Because of plentiful scatterers in the troposphere, microwave can realize a b-LoS propagation. This propagation mechanism is widely known as troposcatter [2-8]. Therefore, EM wave of hostile radar propagated via troposcatter can be utilized for b-LoS location.

Nowadays, passive location system and troposcatter channel have already been well studied [3-7]. To improve the maneuverability of passive troposcatter location system, a receiving antenna mode based on the signal group delay has been proposed [3]. Although this mechanism can conveniently and inexpensively locate targets on the basis of a single station rather than multi-stations, a single station has unreliable detection consequence and limited coverage.
In [4], [5], troposcatter transfer function has been studied and a fading correlation model has been innovatively proposed to analyze the signal received by the array antenna. The multiple signal classification (MUSIC) algorithm has been improved to precisely estimate direction-of-arrival (DOA) of signals received by troposcatter location system [6], [7], however, the coherence of signals are not taken into consideration.

On the basis of available achievements and to provide theoretical basis for designing the passive troposcatter location system, this paper researches on channel characteristics and the challenges for passive troposcatter location system. The chief contributions of this paper are mainly focused on twofold. Troposcatter characteristics including propagation loss, refraction effect and multipath effect are analyzed. On the basis of channel characteristics, maximum operating range is evaluated, the performances of appropriate signal detection, location and DOA estimation are acquired. Through analyzing consequences, research directions are elaborated. The remaining of this paper is organized as follows. Characteristics of troposcatter are described in the next sSection. In Sec. 3, some relevant algorithms combined with simulations are introduced. The conclusions are drawn in Sec. 4.

\section{Characteristics of Troposcatter Link}

Figure 1 displays the principle of passive troposcatter location system. Although scattered power is directed in all directions, segmental troposcatter power can arrive at the passive location system [5-8]. Meanwhile, anti-radiation missile (ARM) has relatively little threat to the passive system. To better cognize the passive troposcatter location system, characteristics of the channel must be studied.

\subsection{Propagation Loss}

Scatter effect leads to a relatively high propagation loss. As a troposcatter link shown in Fig. 1, receiving power can be expressed as [8]

$$
P_{\mathrm{r}}=A P_{\mathrm{t}} G_{\mathrm{t}} G_{\mathrm{r}}\left(\frac{\lambda}{4 \pi}\right)^{2} \int_{V} \frac{g_{1} g_{2} \sigma}{\left(r_{1} r_{2}\right)^{2}} \mathrm{~d} V
$$

where $A$ stands for a factor depending on the statistical distribution of signals, $P_{\mathrm{t}}$ the transmitting power, $\lambda$ the wave- 


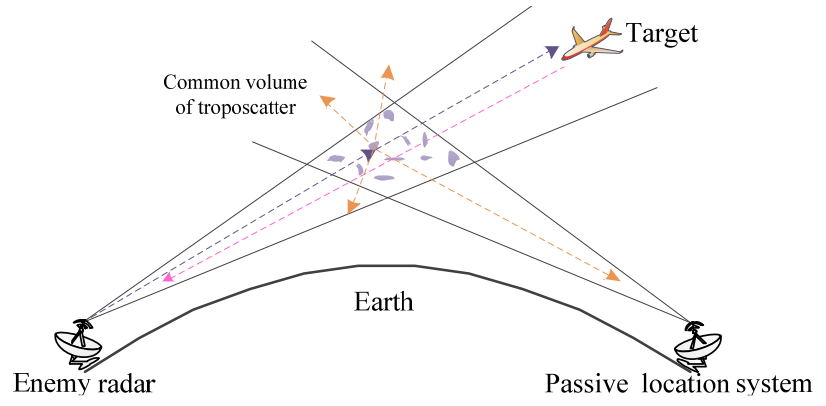

Fig. 1. Passive location system based on troposcatter.

length, $V$ the common volume of two beams, $\sigma$ the scattering cross-section. $G_{\mathrm{t}}, G_{\mathrm{r}}$ denote the plane wave gains of the $\mathrm{TX} / \mathrm{RX}$ antennas, respectively. $r_{1}, r_{2}$ denote the distance between the scatterer to TX/RX antennas, respectively. $g_{1}$, $g_{2}$ represent the directivity function of $\mathrm{TX} / \mathrm{RX}$ antennas, respectively. Some parameters such as $\sigma$ and $V$ are relatively difficult to be estimated. Therefore, several empirical models have been proposed. Zhang method has been accepted internationally as the agreed model. According to Zhang method [8], [9], the computational equation for the main propagation loss $(\mathrm{dB})$ can be given by

$$
\begin{gathered}
L=F+30 \log f+30 \log \Theta+10 \log d+ \\
20 \log (5+\gamma H)+4.34 \gamma h_{0}+L_{\mathrm{c}}
\end{gathered}
$$

where $\Theta$ refers to the scatter angle (mrad), which can be expressed as: $\Theta=\theta_{\mathrm{t}}+\theta_{\mathrm{r}}+1000 \mathrm{~d} / a_{\mathrm{e}}$, here $a_{\mathrm{e}}$ denotes the median effective earth radius. $\theta_{\mathrm{t}}, \theta_{\mathrm{r}}$ are the TX/RX horizon angle, respectively. $F$ denotes the meteorological parameter $(\mathrm{dB}), \gamma$ the atmospheric structure coefficient $\left(\mathrm{km}^{-1}\right)$, the values of them can be determined according a digital map [9]. $f$ refers to the frequency of carrier wave $(\mathrm{MHz}), d$ the distances between the TX/RX antennas $(\mathrm{km}) . H$ denotes the height between the lowest scattering point and the connection between TX/RX antennas $(\mathrm{km})$, which can be expressed as: $H=10^{-3} \theta d / 4$. $h_{0}$ denotes the height between the lowest scattering point and ground $(\mathrm{km})$, which can be expressed as: $h_{0}=10^{-6} \theta^{2} a_{\mathrm{e}} / 8$. The aperture-to-medium coupling loss of an antenna is expressed as $L_{\mathrm{c}}$ [9], which can be calculated as

$$
L_{\mathrm{c}}(\mathrm{dB})=0.07 \exp \left[0.055\left(G_{\mathrm{t}}+G_{\mathrm{r}}\right)\right]
$$

where $\mathrm{G}_{\mathrm{t}, \mathrm{r}}=10 \log \left[4.5(\mathrm{D} / \lambda)^{2}\right], D$ is the diameter of parabolic antenna $(\mathrm{m})$. Gains of array antenna have relationship with arrangement of elements. The high propagation loss varies with several factors including carrier frequency, elevation angle and antenna diameter.

\subsection{Refraction Effect}

The refraction effect resulting from inhomogeneous troposphere cannot only cause a curving path, but also slow down the speed of EM wave. These negative effects can be expressed as path differences and elevation angle errors. Figure 2 shows a geometric mode. B is the scatter point, $\alpha$ the measured angle, $\beta$ the real elevation angle, $\theta$ the geocentric angle, $r_{1}$ the radius of earth, $h_{\theta}$ the altitude of point $\mathrm{B}$,

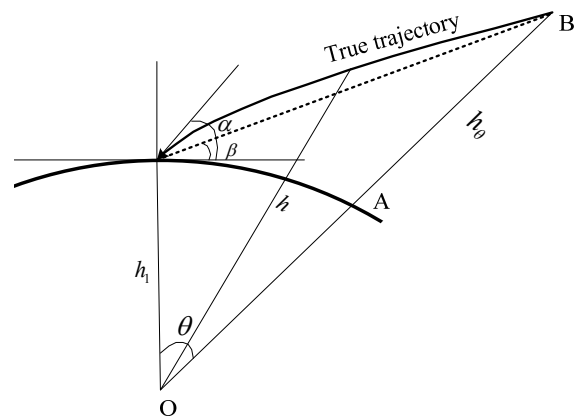

Fig. 2. Geometric model of EM wave.

$r$ the distance between a spot of true trajectory and earth core.

According to the ray tracing method [10], path differences and elevation angle errors can be estimated as

$$
\left\{\begin{array}{l}
\Delta S=\int_{l_{1}}^{l_{2}} \frac{\ln ^{2}(l)}{\sqrt{r^{2} n^{2}(l)-A_{0}^{2}}} \mathrm{~d} r-\sqrt{l_{1}^{2}+l_{2}^{2}-2 l_{1} l_{2} \cos \theta}, \\
\Delta \varepsilon=\alpha-\beta, \\
A_{0}=l_{1} n\left(l_{1}\right) \cos \alpha, \\
l_{2}=l_{1}+h_{\theta}
\end{array}\right.
$$

where $\Delta S$ denote the path differences, $\Delta \varepsilon$ the angle errors, $n(l)$ the refractive index of troposphere. Ray tracing method expresses the geocentric angle as

$$
\theta=n_{0} r_{1} \cos \alpha \int_{l_{1}}^{l_{2}} \frac{\mathrm{d} l}{l \sqrt{n^{2}(l) l^{2}-A_{0}^{2}}}
$$

where $n=1+10^{-6}\left(N_{\text {dry }}+N_{\text {wet }}\right)$. $N_{\text {dry }}, N_{\text {wet }}$ refer to the dry and wet term of the radio refractivity, respectively. The empirical relationship between $N_{\text {dry(wet) }}$ and meteorological parameters can be given by [11]

$$
\left\{\begin{array}{c}
N_{\text {dry }}=77.6 P / T, \\
N_{\text {wet }}=3.73 \times 10^{5} \mathrm{e} / T^{2},
\end{array}\right.
$$

where $P$ denotes the atmospheric pressure in $\mathrm{hPa}, e$ the water vapor pressure in $\mathrm{hPa}, T$ the absolute temperature in $\mathrm{K}$. Above meteorological parameters at any altitude can be measured by a radiosonde balloon. However, the application of radiosonde balloon is limited and inconvenient. To precisely and conveniently calculate $n(r)$, Hopfield model is introduced to improve the ray tracing method. It describes the refractivity as [12]

$$
\begin{gathered}
N_{i}(h)=\frac{N_{i 0}}{H_{i}^{4}}\left(H_{i}-h\right)^{4}, i=\text { dry,wet, } \\
H_{\text {dry }}=40136+148.72\left(T_{0}-273.16\right) \mathrm{m}, \\
H_{\text {wet }}=11000 \mathrm{~m},
\end{gathered}
$$

where $H_{\text {dry }}$ denotes the equivalent height for hydrostatic refractivity, $H_{\text {wet }}$ the equivalent height for wet refractivity. Meteorological parameters of BJFS observation (115.89E, $39.61 \mathrm{~N}$ ) on January 1, 2013 are selected. Figure 3 shows path differences and angle errors estimated through the improved ray tracing method. The higher scatter point and the smaller elevation angle can bring more negative effect. 

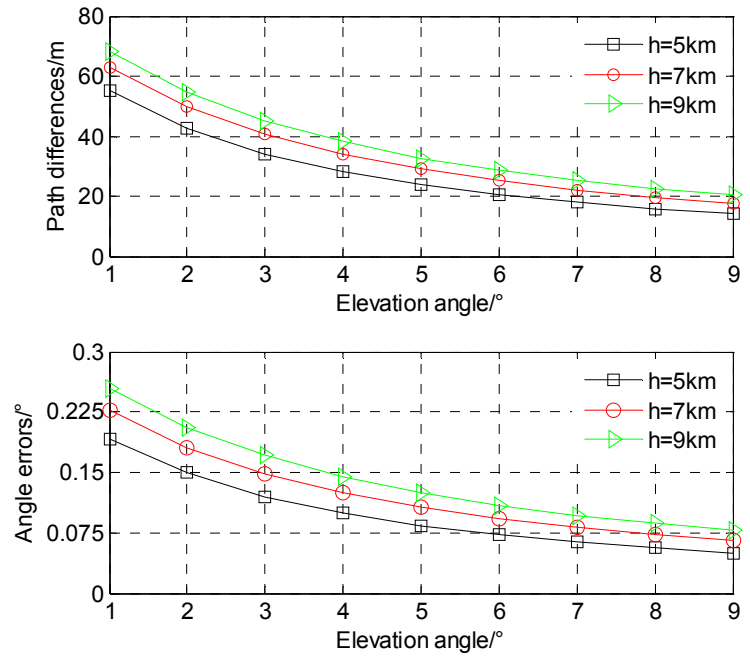

Fig. 3. Errors of EM wave.

\subsection{Multipath Effect}

When EM wave is propagated by troposcatter channel, scattering and reflection effects will be produced as a result of encountering abundant turbulence and scatterers, thus multipath propagation occurs [5], [13], [14]. Multipath propagation will cause multipath fading. The time-frequency coherent characteristics can particularly describe multipath fading. Correlation function can be defined as: $\Gamma\left(\omega_{1}, \omega_{2} ; t_{1}, t_{2}\right)=\left\langle H_{1}\left(\omega_{1}, t_{1}\right) H_{2}^{*}\left(\omega_{2}, t_{2}\right)\right\rangle$, here $H$ is the transfer function. $H$ of troposcatter channel follows [13], [14]

$$
H=B \int_{V} \sqrt{g_{1} g_{2}} \Delta \varepsilon_{\mathrm{r}} \exp \left\{-\mathrm{j}\left[k\left(r_{1}+r_{2}\right)+K \cdot v t\right]\right\} \mathrm{d} V
$$

where $B$ is a constant, $\Delta \varepsilon_{\mathrm{r}}$ the fluctuations of relative permittivity, $k=2 \pi / \lambda$ the wavenumber, $K \cdot v$ the relatively low Doppler frequency shift of scatterer motion. Transfer functions of different EM waves received by the same receiver at different time follows

$$
\left\{\begin{array}{l}
H_{1}=B_{1} \int_{V} \sqrt{g_{1} g_{21}} \Delta \varepsilon_{\mathrm{r}} \exp \left\{-\mathrm{j}\left[k\left(r_{1}+r_{21}\right)+K_{1} \cdot v t_{1}\right]\right\} \mathrm{d} V \\
H_{2}=B_{2} \int_{V} \sqrt{g_{1} g_{22}} \Delta \varepsilon_{\mathrm{r}} \exp \left\{-\mathrm{j}\left[k_{2}\left(r_{1}+r_{22}\right)+K_{2} \cdot v t_{2}\right]\right\} \mathrm{d} V
\end{array}\right.
$$

Since we only care about the frequency coherent characteristics, correlation function can be simplified as $\Gamma\left(\omega_{1}, \omega_{2}\right)$, which can be deduced as

$$
\begin{aligned}
& R_{H 12}=B_{3} \int g_{1} \sqrt{g_{21} g_{22}} \sigma_{v} \\
& \exp \left\{-\mathrm{j}\left[k_{2}\left(r_{1}+r_{22}\right)-k_{2}\left(r_{1}+r_{21}\right)+K_{2} \cdot v \Delta t_{2}-K_{1} \cdot v \Delta t_{1}\right]\right\} \mathrm{d} V .
\end{aligned}
$$

After a series of deduction, the fading correlation coefficient follows [8], [14]

$$
\rho_{\mathrm{H}}(\Delta f) \approx\left\{-\left(\frac{\Delta f}{\mathrm{~F}_{\mathrm{H}}}\right)^{2}-\mathrm{jI}_{\mathrm{f}} \Delta f\right\}
$$

where $\rho$ denotes the correlation coefficient, $\Delta f$ the frequency difference of received signals. $\mathrm{F}_{\mathrm{H}}$ and $\mathrm{I}_{\mathrm{f}}$ can be given by

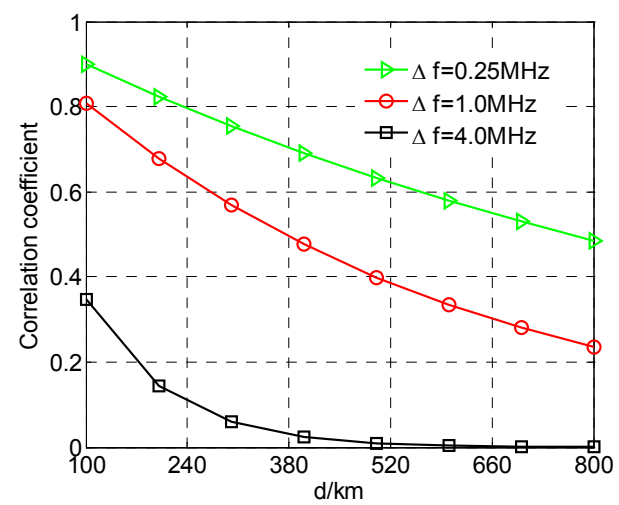

Fig. 4. Correlation coefficient of EM wave.

$$
\left\{\begin{array}{c}
\mathrm{F}_{\mathrm{H}}=\frac{4 c \sqrt{\ln 2}}{\left(1+s_{2}\right) \pi H \sqrt{\left(s_{1} \psi_{v 1}\right)^{2}+\psi_{v 2}^{2}}}, \\
\mathrm{I}_{\mathrm{f}}=2 \pi \tau_{0}-\frac{\pi d}{2 c \sqrt{\ln 2}}\left(\Theta_{20} \psi_{v 1} e_{1}+\Theta_{10} \psi_{v 2} e_{2}\right) .
\end{array}\right.
$$

In (12), $\Theta_{10}\left(\Theta_{20}\right)$ denotes the lowest horizontal beam elevation, $\tau_{0}$ the time delays of shortest path. $e_{1} / e_{2}<<1$, $s_{2}=1 / s_{1}=\Theta_{10} / \Theta_{20}$, beamwidth of the parabolic antenna can be generally given as $\psi=1.2 \lambda / D[14]$. For array antenna, it depends on the beamforming method.

Figure 4 shows the correlation coefficient with parameters: $D_{\mathrm{t}}=10 \mathrm{~m}, D_{\mathrm{r}}=5 \mathrm{~m}, f=5 \mathrm{GHz}, \Theta_{10}=\Theta_{20}=1^{\circ}$. The signals with high correlation may exist, larger frequency difference and longer distance can lead to lower correlation. Cooperative system can change their parameters to avoid the negative fading effect. Different from the cooperative system, passive location system is incapable of action to change this situation.

\section{Performance of Passive System}

In this section, performance of passive system including maximum operating range, signal detection, location and DOA estimation is analyzed.

\subsection{Maximum Operating Range}

To get maximum operating range, we assume that the location system has an optimal azimuth. Then, power received by a location system can be written as

$$
P_{\mathrm{r}}=P_{\mathrm{t}}-L+G_{\mathrm{t}}+G_{\mathrm{r}}
$$

where $P_{\mathrm{t}}$ is the transmitting power of an early-warning radar. Only $P_{\mathrm{r}} \geq S$, the system can work normally. Here, $S$ denotes the receiver sensitivity. $P_{\mathrm{r}}$ has relationship with the distance between target and location system, when $P_{\mathrm{r}}=S$, the location system can realize the maximum operating range. Therefore, we can estimate the maximum range through solving equation: $P_{\mathrm{r}}=S$. Precipitation affects many wireless links, rain has obvious effect on the troposcatter link [14-16]. Many rain attenuation models for satellite communication (SATCOM) have been proposed. The most 


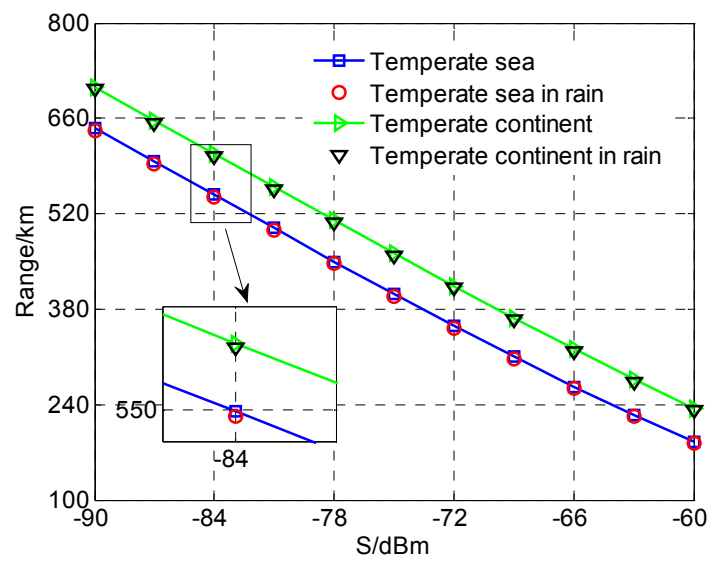

Fig. 5. Maximum operating range changing with $S$.

difference between SATCOM and troposcatter lies in propagation path. In view of different places of scatter point, a reasonable method for calculating the path length is introduced in [17]. Figure 5 presents the maximum operating range changing with $S$ under channel parameters: $f=5 \mathrm{GHz}, D_{\mathrm{t}}=10 \mathrm{~m}, D_{\mathrm{r}}=5 \mathrm{~m}, \theta_{\mathrm{t}}=1^{\circ}, P_{\mathrm{t}}=25 \mathrm{~kW}$, rain rate is $30 \mathrm{~mm} / \mathrm{h}$, path length in rain cell is $20 \mathrm{~km}$.

From Fig. 5, the passive troposcatter location system can realize b-LoS location, operating range decreases with $S$ growing up. If the system wants to realize a longer operating range, the receiver must have a lower sensitivity. Array antenna which has high gains and wide spatial coverage can be used to capture weak scattering signal, thus the passive system equipped with array antenna has a large operating range and coverage.

\subsection{Signal Detection}

Only signal detection algorithm makes a faultless decision, the next works will be carried out. To locate the enemy radar, passive system always has distributed stations. Distributed system has a large coverage and inherent superiority at realizing cooperative signal detection (CSD), which can resist the undesirable shadow and multipath fading [18-21]. Each terminal node of passive system can be regarded as a fusion center (FC). Local decision can be delivered by the reporting channel. According to decision fusion, $u_{i}$ stands for the decision of node $i$, here $u_{i}=1$ for the decision of present signal and $u_{i}=0$ for the decision of absent signal. FC makes a global decision based on data fusion or decision fusion. The former consumes more wireless resources. Performance of the latter could be improved remarkably by increasing the number of nodes. According to the OR-rule, FC can make a global decision as

$$
\left\{\begin{array}{c}
Q_{\mathrm{FA}}=1-\prod_{i=1}^{L}\left[\left(1-P_{\mathrm{FA}}\right)\left(1-P_{\mathrm{e}}\right)+P_{\mathrm{FA}} P_{\mathrm{e}}\right], \\
Q_{\mathrm{D}}=1-\prod_{i=1}^{L}\left[\left(1-P_{\mathrm{D}}\right)\left(1-P_{\mathrm{e}}\right)+P_{\mathrm{D}} P_{\mathrm{e}}\right]
\end{array}\right.
$$

where $Q_{\mathrm{FA}}$ stands for the global probability of false alarm, $Q_{\text {D }}$ the global probability of detection, $L$ the number of terminal nodes, $P_{\mathrm{FA}}$ the terminal probability of false alarm, $P_{\mathrm{D}}$ the terminal probability of detection, $P_{\mathrm{e}}$ the bit error rate (BER) of reporting channel. Detection problem can be transformed into a binary hypothesis test, which can be presented as

$$
\begin{cases}H_{0}: r[n]=w[n] & n=0,1, \cdots, N-1, \\ H_{1}: r[n]=s[n]+w[n] & n=0,1, \cdots, N-1\end{cases}
$$

where $H_{0}$ refers to the absence of a signal, $H_{1}$ the presence of a signal, $s[n]$ the unknown deterministic signal, $w[n]$ the additive white Gauss noises (AWGN) with zero mean and $\sigma^{2}$ variance. Current signal detection algorithms mainly focus on matched filter detection (MFD), cyclostationary feature detection (CFD) and energy detection (ED). Different from cooperative occasions, passive system probably lacks priori knowledge of the targets. ED which has simpler implementation and less requirement of priori knowledge is a preferred candidate for passive system. According to $\mathrm{ED}$, if $Y>\tau, s[n]$ is present, otherwise the signal is absent. Here, $Y$ denotes the observed energy, $\tau$ the predefined threshold. ED gives $P_{\mathrm{FA}}$ and $P_{\mathrm{D}}$ as [19]

$$
\left\{\begin{array}{c}
P_{\mathrm{FA}}=\operatorname{Pr}\left(Y>\tau \mid H_{0}\right)=Q\left(\frac{\tau-\sigma^{2}}{\sigma^{2} \sqrt{2 / M}}\right), \\
P_{\mathrm{D}}=\operatorname{Pr}\left(Y>\tau \mid H_{1}\right)=Q\left(\frac{\tau / \sigma^{2}-(1+\gamma)}{\sqrt{2 / M}(1+\gamma)}\right) .
\end{array}\right.
$$

Troposcatter can be approximated as a Rayleigh fading channel, probability distribution function (PDF) of average SNR follows [20]

$$
f(\gamma)=(1 / \bar{\gamma}) \exp [-\gamma / \bar{\gamma}]
$$

where $\bar{\gamma}$ refers to average SNR. Therefore, $P_{\mathrm{D}}$ can be given by

$$
P_{\mathrm{D}}=\int_{0}^{\infty} Q\left(\frac{\tau / \sigma^{2}-(1+\gamma)}{\sqrt{2 / M}(1+\gamma)}\right) f(\gamma) \mathrm{d} \gamma .
$$

In conventional ED, an unstable environment can lead to varying noises, double-threshold ED (DTED) can efficiently coping with varying noises [21]. Thresholds of DTED follow

$$
\left\{\begin{array}{l}
\lambda_{1}=\sqrt{\frac{2}{M} Q^{-1}\left(P_{\mathrm{f}}\right)+1} \frac{1}{\rho} \sigma^{2}, \\
\lambda_{2}=\sqrt{\frac{2}{M} Q^{-1}\left(P_{\mathrm{f}}\right)+1} \rho \sigma^{2}
\end{array}\right.
$$

where $\rho$ denotes the uncertainty parameter of noises, it can be expressed as

$$
\rho=\frac{\sigma_{u}^{2}}{\sigma^{2}} \in\left[10^{-A / 10}, 10^{A / 10}\right], A \geq 0
$$

where $A$ refers to the uncertainty factor. 


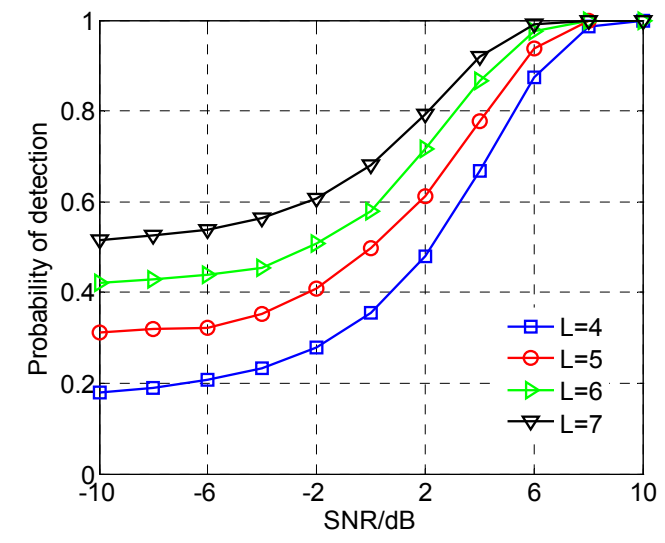

Fig. 6. $P_{\mathrm{D}}$ of CSD.

Figure 6 shows the $P_{\mathrm{D}}$ changing with $\mathrm{SNR}$ with the parameter: $P_{\mathrm{FA}}=0.01, \sigma^{2}=1, A=1, P_{\mathrm{e}}=10^{-4}$. Higher SNR and larger number of terminal nodes can result in a better performance. As known, troposcatter has a relatively high propagation loss and the received signal suffers from low SNR. As well, when observed energy lies between the two thresholds, no decision is made. Therefore, signal detection algorithm with a good performance is in urgent need. Several methods have been proposed to overcome this detection confusion [21-25]. The chief improvements on detection confusion focus on two aspects. On the one hand, each node sends its observed energy to FC, and FC makes a global decision based on terminal observed energy.

On the other hand, each node independently overcomes its detection confusion, FC makes global decision based on terminal decision. In [21], double-threshold cooperative detection scheme based on weighed combination is proposed. FC can make a global decision based on weighed data delivered by reporting channel. Because FC applies more data to make global decision, reporting channel with more bandwidth is required, and threshold employed by FC to make decision is difficult to be determined. Same defects also exist in [22], [23]. In [24], [25], confusion area is equally divided into four parts. When observed energy lies within confusion region, terminal node sends 2-bit decision. This mechanism can overcome the confusion area without increasing the burden on reporting channel. However, dividing confusion area with the same width lacks generality. Meanwhile, time synchronization is the prerequisite of coordinating distributed system operations [26]. During operating the passive system, time synchronization with high precision must be got and kept.

\subsection{Location and DOA Estimation}

The radiation source can be located on the basis of time differences of arrival (TDOA) and elevation angle. Analyzed in Sec. 2.2, troposcatter has negative effect on estimating elevation angle and path length, which can contribute obvious drawbacks to this location algorithm. Moreover, it is difficult to estimate the altitude of scatter point, as well as path length.

Figure 7 displays a location algorithm only depending on azimuth. Appropriate DOA algorithm can precisely esti-

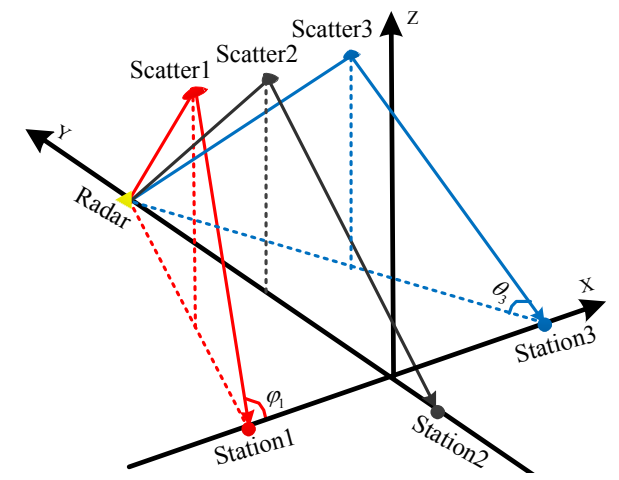

Fig. 7. Diagram of location algorithm.

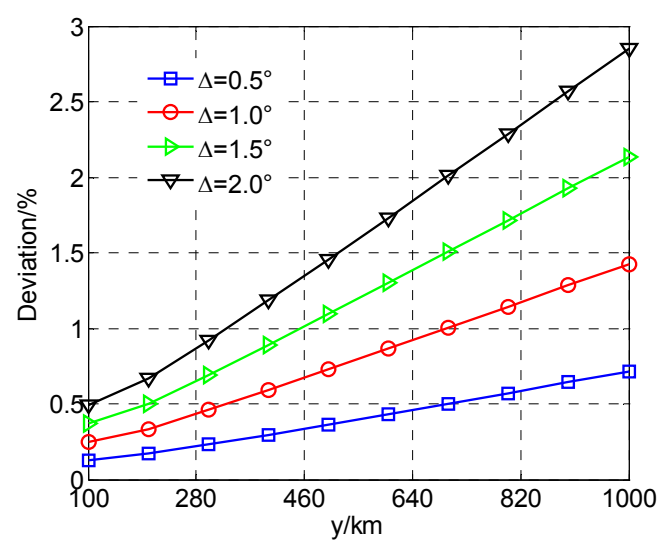

Fig. 8. GDOP variation with azimuth bias.

mate $\theta$ and $\varphi$. As known, the effect on $\theta$ caused by inhomogeneous troposphere is more apparent than $\varphi$. Coordinate of hostile radar and detection station is $(x, y, z)$ and $\left(x_{i}, y_{i}, z_{i}\right)$, respectively. The relationship between them can be expressed as

$$
\tan \varphi_{i}=\frac{\sqrt{\left(z-z_{i}\right)^{2}+\left(y-y_{i}\right)^{2}}}{x-x_{i}}, i=1,2,3 .
$$

We can obtain the location of radar following (21). It requires a high precision $\varphi$, which depends on an appropriate DOA algorithm. The typical targets of passive troposcatter location system are the radiations with low speed or stationary location, such as ground-based radars and ship-borne radars. Therefore, estimating altitude of targets is not vital, while their longitude and latitude are the important factors. Geometric dilution of precision (GDOP) [27] is employed to evaluate the location algorithm. Coordinates of two passive stations are $(0 \mathrm{~km}, 0 \mathrm{~km})$ and $(0 \mathrm{~km}, 100 \mathrm{~km})$, the location bias of observation is $10 \mathrm{~m}$. Coordinate of target is $(0 \mathrm{~km}, y \mathrm{~km})$. Figure 8 depicts the GDOP varying with azimuth bias. Here, $\Delta$ denotes the azimuth bias, ordinate denotes the value of relative deviation following GDOP dividing $y$.

From Fig. 8, the bias of azimuth decides the location performance of passive system. Therefore, DOA estimation algorithm with high performance is vital. Array antenna can realize more precise DOA estimation than parabolic antenna. Many algorithms based on array antenna have been developed over years, such as multiple signal classifi- 
cation (MUSIC), estimation of signal parameters via rotation invariance technique (ESPRIT) [6], [7], [28-30].

When $K$ far-field sources arrive at an $M$-element uniform linear array (ULA) with arrival angle $\theta_{i}(i=1,2, \ldots, K)$, the array output follows

$$
\mathbf{X}(t)=\mathbf{A}(\theta) \mathbf{S}(t)+\mathbf{N}(t), \quad t=1,2, \cdots, n
$$

where $\mathbf{N}(t)$ is the noises vector. $\mathbf{A}(\theta)$ can be given by $\mathbf{A}(\theta)=\left[\mathbf{a}\left(\theta_{1}\right), \mathbf{a}\left(\theta_{2}\right), \ldots, \mathbf{a}\left(\theta_{K}\right)\right]$, here $\mathbf{a}\left(\theta_{i}\right)$ can be expressed as

$$
\mathbf{a}\left(\theta_{i}\right)=\left[1, \mathrm{e}^{-\mathrm{j} \frac{2 \pi}{\lambda_{0}} d \sin \left(\theta_{i}\right)}, \cdots, \mathrm{e}^{-\mathrm{j} \frac{2 \pi}{\lambda_{0}}(M-1) d \sin \left(\theta_{i}\right)}\right]^{\mathrm{T}} .
$$

Covariance matrix can be $\mathbf{R}=\mathrm{E}\left[\mathbf{X}(t) \mathbf{X}^{\mathrm{H}}(t)\right]=$ $\mathbf{A} \mathbf{R}_{\mathrm{S}} \mathbf{A}^{\mathrm{H}}+\mathbf{R}_{\mathrm{N}}$, here $\mathbf{R}_{\mathrm{S}}$ is the signal covariance matrix, $\mathbf{R}_{\mathrm{N}}$ the noises covariance matrix. As a result of finite samples, it can be approximately calculated as [28]

$$
\hat{\mathbf{R}}=\frac{1}{n} \sum_{t=1}^{n} \mathbf{X}(t) \mathbf{X}^{\mathrm{H}}(t)
$$

where $n$ is the snapshots. The covariance matrix can be decomposed to form the signal and noise subspaces. MUSIC has a wide application and estimates DOA according to orthogonality of the two subspaces [29]. Because highly correlated and even coherent signals can deteriorate the orthogonality of subspaces, MUSIC will be invalid under this situation. As analyzed in Sec. 2.3, multipath propagation may make the sources partially correlated and even coherent. Furthermore, active decoying technique setting one or more additional radiant baits near the true radiant source can enhance its survivability. The deceptive signals transmitted by baits are always coherent with the true signals. Therefore, DOA estimation algorithms must process the uncorrelated, highly correlated and even coherent signals concurrently received by the passive system. Spatial smoothing algorithm becomes a common method of coherent sources pretreatment [30].

Figure 9 displays the rule to determine subarrays. $L$ denotes the number of subarrays. Each subarray has $m$ elements, then $m=M-L+1$. The covariance matrix after forward spatial smoothing (FSS) follows

$$
\mathbf{R}_{\mathrm{F}}=\frac{1}{L} \sum_{k=1}^{L} \mathbf{F}_{k} \mathbf{R F}_{k}^{\mathrm{T}}
$$

where $\mathbf{F}_{k}=\left[\mathbf{0}_{m \times(k-1)}\left|\mathbf{I}_{m}\right| \mathbf{0}_{m \times(N-k-m+1)}\right]$. The covariance matrix after forward and backward spatial smoothing (FBSS) follows [26]

$$
\mathbf{R}_{\mathrm{FB}}=\frac{1}{2 L} \sum_{k=1}^{L} \mathbf{F}_{k}\left(\mathbf{R}+\mathbf{J R}^{*} \mathbf{J}\right) \mathbf{F}_{k}^{\mathrm{T}}
$$

where $*$ denotes conjugate operation, the elements of the anti-diagonal of $\mathbf{J}$ are 1 .

The classical MUSIC can employ the consequences of spatial smoothing to estimate DOA. Figure 10 displays the

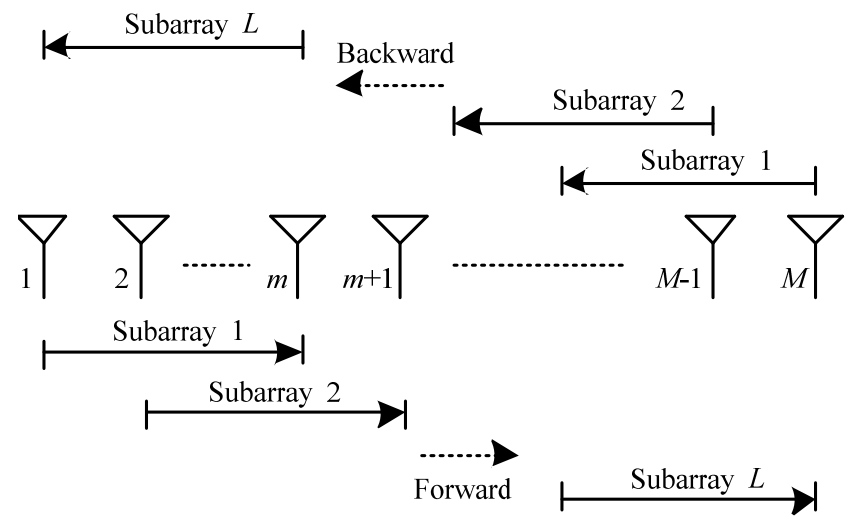

Fig. 9. Forward/backward spatial smoothing scheme.

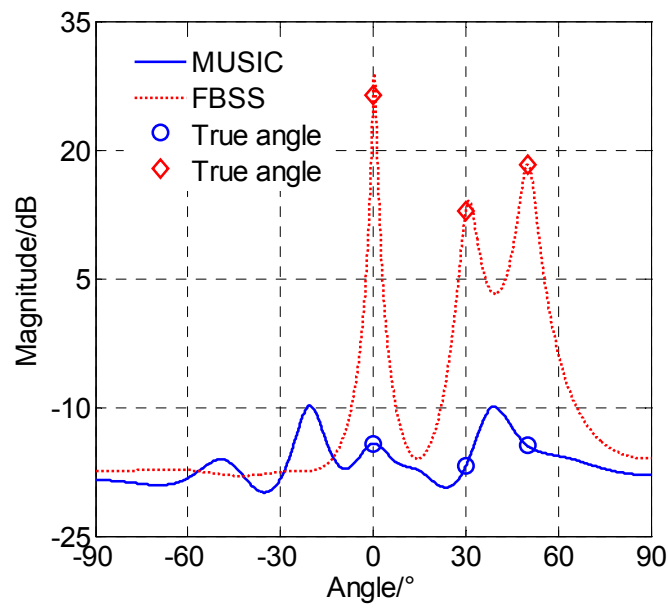

Fig. 10. Estimation performance of DOA algorithms.

estimation performance. In this simulation, the true DOA of three signals is $\left(0^{\circ}, 30^{\circ}, 50^{\circ}\right)$, SNR is $(4 \mathrm{~dB},-1 \mathrm{~dB}, 0 \mathrm{~dB})$, number of snapshots is $300, M=10, m=3$. The first two signals are coherent, and the third signal is uncorrelated with others. As shown in Fig. 10, after FBSS, the DOA can be precisely estimated by MUSIC algorithm. And the coherent signals make the conventional MUSIC algorithm invalid. Though spatial smoothing algorithm has a good performance, it obviously reduces the array aperture, the maximum number of resolved sources will be restricted.

Similarly, maximum likelihood (ML) methods can also resolve the coherent sources directly, but they need multidimensional search and thus suffer from burdensome computation. Some other algorithms can separately process the uncorrelated and coherent sources. They can resolve more sources in most situations. But the main disadvantage of such methods is large computational burden and difficult to eliminate the contributions of different sources. Future DOA estimation algorithm used in passive troposcatter location system must efficiently work under circumstance that the uncorrelated and coherent sources coexist. Meanwhile, some arrays based on new structures or new systems are put forward, such as nested array, coprime array, etc. Some of them can process the coherent signals based on new technologies. 


\section{Conclusions}

In this paper, to provide theoretical basis for passive troposcatter location system, the channel characteristics including propagation loss, refraction effect and multipath fading are discussed. In addition, maximum operating range, signal detection, location and DOA estimation are studied on the basis of channel characteristics. According to simulation results, passive system has a b-LoS coverage, energy detection can cope with the received signal without priori knowledge, the accuracy of location algorithm based on azimuth can be accepted, spatial smoothing is an effective de-correlation algorithm. At the same time, defects of available algorithms and future directions for developing passive troposcatter location system are suggested.

\section{Acknowledgment}

We gratefully acknowledge anonymous reviewers who read and make insightful and constructive suggestions. This work was supported by the National Natural Science Foundation of China under grant No. 61671468 and 61701525. And it also was supported by the Postdoctoral Science Foundation of China under grant No. 2017M623351. In addition, Zan LIU wants to thank the support from Jiao QIU over the past years.

\section{References}

[1] YANG, F., XU, Q., LI, B. Ship detection from optical satellite image based on saliency segmentation and structure-LBP feature. IEEE Geoscience and Remote Sensing Letters, 2017, vol. 14, no. 5, p. 602-606. DOI: 10.1109/LGRS.2017.2664118

[2] DINC, E., AKAN, O. B. More than the eye can see: coherence time and coherence bandwidth of troposcatter links for mobile receivers. IEEE Vehicular Technology Magazine, 2015, vol. 10, no. 2, p. 86-92. DOI: $10.1109 / \mathrm{mvt} .2015 .2410786$

[3] ZHUANG WANG, MENGNAN WANG, QI WANG, et al. Receiving antenna mode of troposcatter passive ranging based on the signal group delay. IET Microwaves, Antennas \& Propagation, 2017, vol. 11, no. 1, p. 121-128. DOI: 10.1049/iet-map.2016.0242

[4] MENGNAN WANG, ZHUANG WANG, JING WANG, et al. Fading correlation modelling for troposcatter microwave propagation in array antenna detection applications. IET Microwaves, Antennas \& Propagation, 2017, vol. 11, no. 6, p. 833-843. DOI: 10.1049/iet-map. 2016.0229

[5] MENGNAN WANG, ZHUANG WANG, ZHU CHENG, et al. Target detection for a kind of troposcatter over-the-horizon passive radar based on channel fading information. IET Radar, Sonar \& Navigation, 2018, vol. 12, no. 4, p. 407-416. DOI: 10.1049/ietrsn.2017.0435

[6] QI WANG, ZHU CHENG, ZHUANG WANG, et al. The troposcatter array signal receiving model and processing algorithm. In 12th International Conference on Signal Processing (ICSP2014). Hangzhou (China), 2014, p. 283-287. DOI: 10.1109/ICOSP.2014.7015013

[7] JING WANG, ZHUANG WANG, MENGNAN WANG, et al. Time-frequency diffusion analysis for DOA estimation of tropospheric scatter signal. In 2016 Progress In Electromagnetic Research Symposium (PIERS). Shanghai (China), 2016, p. 3547-3552. DOI: $10.1109 /$ piers.2016.7735369
[8] MINGGAO ZHANG. Troposcatter Propagation. Beijing: Publishing House of Electronic Industry, 2004.

[9] LEI LI, ZHENSEN WU, LEKE LIN, et al. Study on the prediction of troposcatter transmission loss. IEEE Transactions on Antennas and Propagation, 2016, vol. 64, no. 3, p. 1071-1079. DOI: 10.1109/TAP.2016.2515125

[10] QINGLIN ZHU, ZHENWEI ZHAO, LEKE LIN. Real time estimation of slant path tropospheric delay at very low elevation based on singular ground-based global positioning system station. IET Radar, Sonar and Navigation, 2013, vol. 7, no. 7, p. 808-814. DOI: 10.1049 /iet-rsn.2012.0235

[11] SHUHONG GONG, DAOPU YAN, XUAN WANG. A novel idea of purposefully affecting radio wave propagation by coherent acoustic source-induced atmospheric refractivity fluctuation. Radio Science, 2016, vol. 50 no. 10, p. 983-996. DOI: $10.1002 / 2015 \mathrm{rs} 005660$

[12] HOPFIELD, H. S. Two-quartic tropospheric refractivity profile for correcting satellite data. Journal of Geophysical Research, 1969, vol. 74, no. 18, p. 4487-4499. DOI: 10.1029/JC074i018p04487

[13] DINC, E., AKAN, O. B. Fading correlation analysis in MIMOOFDM troposcatter communications: space, frequency, angle and space-frequency diversity. IEEE Transactions on Communications, 2015, vol. 63, no. 2, p. 476-486. DOI: 10.1109/TCOMM.2014.2387159

[14] CHENGLONG LI, XIHONG CHEN, ZEDONG XIE. A closedform expression of coherence bandwidth for troposcatter links. IEEE Communications Letters, 2018, vol. 22, no. 3, p. 646-649. DOI: 10.1109/LCOMM. 2017.2785850

[15] International Telecommunication Union (ITU) Recommendation P.835-5. Reference Standard Atmospheres. Geneva, Switzerland, 2012.

[16] CHENGLONG LI, XIHONG CHEN, XIAOPENG LIU. Cognitive tropospheric scatter communication. IEEE Transactions on Vehicular Technology, 2018, vol. 67, no. 2, p. 1482-1491. DOI: 10.1109/TVT.2017.2761440

[17] DINC, E., AKAN, O. B. A non-uniform spatial rain attenuation model for troposcatter communication links. IEEE Wireless Communications Letters, 2015, vol. 4, no. 4, p. 441-444. DOI: 10.1109/LWC.2015.2433261

[18] HACHEMI, M. H., FEHAM, M., ADARDOUR, H. E. Predicting the probability of spectrum sensing with LMS process in heterogeneous LTE networks. Radioengineering, 2016, vol. 25, no. 4, p. 808-820. DOI: 10.13164/re.2016.0808

[19] BAE, S., SO, J., KIM, H. On optimal cooperative sensing with energy detection in cognitive radio. Sensors, 2017, vol. 17, no. 9, p. 1-15. DOI: $10.3390 / \mathrm{s} 17092111$

[20] WEBJING YUE, BAOYU ZHENG, QIINGMIN MENG, et al. Robust cooperative spectrum sensing schemes for fading channels in cognitive radio networks. Science China (Information Sciences), 2011, vol. 54, no. 2, p. 348-359. DOI: 10.1007/s11432-010-4147-X

[21] XIN LIU, CHENGWEN, ZHANG, XUEZHI TAN. Doublethreshold cooperative detection for cognitive radio based on weighing. Wireless Communications and Mobile Computing, 2015, vol. 14 , no. 13 , p. $1231-1243$. DOI: $10.1002 / \mathrm{wcm} .2219$

[22] SHIQI LIU, CHONGQIAN CHEN, HAIHONG LI, et al. Double thresholds energy detection based cooperative spectrum sensing for cognitive radios. Chinese Journal of Radio Science, 2013, vol. 28 , no. 3 , p. 420-424. (In Chinese)

[23] JIANG ZHU, ZHENGGUANG XU, FURONG WANG, et al. Double threshold energy detection of cooperative spectrum sensing in cognitive radio. In 2008 3rd International Conference on Cognitive Radio Oriented Wireless Networks and Communications (CrownCom 2008). Singapore, May, 2008. DOI: 10.1109/CROWNCOM.2008.4562451 
[24] PANKAJ VERMA, BRAHMJIT SINGH. Overcoming sensing failure problem in double threshold based cooperative spectrum sensing. Optik-International Journal for Light and Electron Optics, 2016, vol. 127, p. 4200-4204. DOI: 10.1016/j.ijleo.2016.01.108

[25] SHIQI LIU, BINJIE HU, XIANYI WANG. Hierarchical cooperative spectrum sensing based on double thresholds energy detection. IEEE Communications Letters, 2012, vol. 16, no. 7, p. 1096-1099. DOI: 10.1109/LCOMM.2012.050112.120765

[26] ZAN LIU, XIHONG CHEN, JIN LIU, et al. High precision clock bias prediction model in clock synchronization system. Mathematical Problems in Engineering, 2016, Article ID 1813403 , p. 1-6. DOI: $10.1155 / 2016 / 1813403$

[27] BI LIU, YUNLONG TENG, QI HUANG. GDOP minimum with satellites in the single-point positioning. Advances in Space Research, 2017, vol. 60, no. 7, p. 1400-1403. DOI: 10.1016/j.asr.2017.06.049

[28] HUAXIN YU, XIAOFEI ZHANG, XUEQIANG CHEN, et al. Computationally efficient DOA tracking algorithm in monostatic MIMO radar with automatic association. International Journal of Antennas and Propagation, 2014, Article ID 501478, p. 1-10. DOI $10.1155 / 2014 / 501478$

[29] KAIBO CUI, XI CHEN, JINGIAN HUANG, et al. DOA estimation of multiple LFM sources using a STFT-based and FBSS-based MUSIC algorithm. Radioengineering, 2017, vol. 26, no. 4, p. 1126-1137. DOI: 10.13164/re.2017.1126

[30] SHAIKH, S. A., TONELlO, A. M. DOA estimation in EM lens assisted massive antenna system using subsets based antenna selection and high resolution algorithms. Radioengineering, 2018, vol. 27 , no. 1, p. 159-168. DOI: 10.13164/re.2018.0159

\section{About the Authors ...}

Zan LIU was born in Hebei province, People's Republic of China. He received the B.S. and M.S. degrees in 2013 and 2015, respectively, from the Air Force Engineering University, Xi'an. He is currently working toward the $\mathrm{PhD}$ degree in the Air and Missile Defense College. His research interests include time synchronization and passive detection.

XiHong CHEN was born in Shaanxi province, People's Republic of China. He received his B.S. and $\mathrm{PhD}$ degrees from the Missile Institute of Air Force Engineering University, Xi'an, China, in 1982 and 2010, respectively, and his M.S. degree from the Xi'an Electronic Science and Technology University, Xi'an, China, in 1992. He is now a professor of the Air Force Engineering University. His current interests are the tactical high capacity troposcatter communication system and time synchronization technology.

Qiang LIU was born in Hunan province, People's Republic of China. He received the M.S. and $\mathrm{PhD}$ degrees in 2010 and 2014, respectively, from the Air Force Engineering University, Xi'an. He is currently working at postdoctoral workstation in the Air and Missile Defense College. His research interests include time synchronization and passive detection. 